\title{
A Novel Resource Allocation Scheme for Reducing MAP Overhead and Maximizing Throughput in MIMO-OFDM Systems
}

\author{
Chung Ha Koh, Kyung Ho Sohn, Ji Wan Song, and Young Yong Kim \\ Dept. of Electrical and Electronic Engineering, \\ Yonsei University, \\ Seoul, Korea 120-749 \\ \{ski244, heroson7, wanbabo, y2k\}@yonsei.ac.kr
}

\begin{abstract}
We propose a novel resource allocation scheme, which can reduce MAP overhead and maximize the throughput in the MIMO-OFDM systems. In the message based broadband access system, we need to minimize the MAP overhead since the excessive MAP overhead causes degradation of system throughput. Increasing the size of resource allocation unit can reduce MAP overhead. However, multiuser diversity gain becomes smaller as the size of re-source allocation unit increases. Therefore, we investigate joint optimization between multiuser diversity gain and MAP overhead size. Using the proposed scheme, we can reduce MAP overhead size as well as achieve high throughput.
\end{abstract}

\section{Introduction}

Message based multiplexing is becoming more prominent than channel based multiplexing in the next generation communication systems like 802.16 Broadband Wireless Network [1]. The system operating with channel based multiplexing gives user monopolistic rights to use the channel resource when a session is opened. Channel based multiplexing employs predivided area as the resource allocation unit. In contrast, the system exploiting message based multiplexing allocates the resource using flexible resource allocation unit. Message based multiplexing is well matched with the burst nature of data traffic, and it makes the system use radio resource effectively.

Message based multiplexing system has to inform all users of the results from resource allocation by using a frame message because the results of resource allocation are changed every frame. However, message based multiplexing has very large message overhead since every user is given all information pertaining to the allocation results. In the Broadband Wireless Network system, the base station (BS) broadcasts a MAP message which is appended to the front part of each frame in order to transfer the allocation information. Furthermore, the users are needed to receive the MAP message reliably, so the MAP message must be transmitted with low order modulation and heavy coding. Therefore, the transmission time of the MAP message becomes longer and system throughput reduces relatively with the decrease of data transmit time. 
Our main focus is on the allocation unit of frequency resource. We assume that a subband consists of several subchannels and regard it as the frequency resource allocation unit. Note that the subband size means the number of subchannels in it. When the subband size becomes larger, the MAP overhead size becomes smaller since the number of allocation is decreased. On the other hand, as the allocation unit increases, the multiuser diversity gain decreases because the capacity of a subband is determined by minimum channel capacity of subchannels in it. Therefore the potential to exploit higher data throughput introduces a trade-off problem between the size of the MAP message and multiuser diversity gain.

After analyzing the relation between multiuser diversity gain and MAP overhead size, we propose a new scheme of determining an optimum resource allocation unit. In this paper, we have found the optimum subband size to maximize the system through-put. Our simulation results shows that the proposed algorithm tends to outperform the throughput in terms of maximizing throughput.

The rest of this paper is begins with the performance analysis of trade-off between multiuser diversity gain and MAP overhead. Section 3 shows simulation results, and conclusions are drawn with some final remarks in Section 4.

\section{Problem Formulation and Analysis}

In this section, we find the optimum subband size which maximizes the system throughput considering trade-off relationship between multiuser diversity gain and MAP overhead size. Firstly, to simplify our analysis we assume that the all subchannels have an independent and identical distribution (i.i.d.) channel capacity. Then, we expand the results to the situation in which each user has different channel capacity and propose the heuristic algorithm for that case.

\subsection{I.I.D. Channel Capacity Case}

To simply our analysis, we consider i.i.d. subchannel capacity distribution and best user selection scheduling scheme. Let us assume $X_{k, n}$ is an i.i.d. sequence of random variable for the capacity given subchannel $n$, user $k$. If we make a subband by binding $p$ subchannels and use it as a unit of resource allocation, $Y_{k}$, a subband capacity of user $k$, is

$$
Y_{k}=\operatorname{mean}\left\{X_{k, 1}, X_{k, 2}, \cdots, X_{k, p}\right\}
$$

$X$ has a Gaussian distribution under the condition of sufficient number of antennas in the MIMO system [2],[5], so the average value of an $X$ sequences and $Y$ have also a Gaussian distribution. If the mean value of $X$ is $\mu_{X}$ and the variance value is $\sigma_{X}^{2}$, then the mean value of $Y$, denoted as $\mu_{Y}$, is $\mu_{X}$, and the value of variance $Y$, denoted as $\sigma_{Y}^{2}$, is $\left(\sigma_{X} / p\right)^{2}$. When we consider the best user selection scheduling that allocates the subband to maximum subband capacity user, the average throughput is calculated as follows [5].

$$
M=\max \left\{Y_{1}, Y_{2}, \cdots, Y_{K}\right\}
$$




$$
E[M]=\mu_{Y}+\sqrt{2 \sigma_{Y}^{2} \log K}=\mu_{X}+\frac{\sqrt{2 \sigma_{X}^{2} \log K}}{p}
$$

Here, $\sqrt{2 \sigma_{X}^{2} \log K} / p$ is the multiuser diversity gain from scheduling leading to high increase of throughput. When the value of $p$ gets larger, the amount of multiuser diversity gain gets smaller. This is because selecting max capacity in each subchannel can obtain larger scheduling gain than selecting max capacity among the mean capacity of several binding subchannels.

The transmission time for the MAP message is formulated as follows. Downlink MAP message consist of various elements [1]. The DL-MAP is specified by the MAP IE, which represents the allocating result that exploit the same modulation order and coding rate. MAP message transmission time depends on the number of MAP information elements (IEs) which indicate the number of allocated users in a frame.

When we allocate resources in subbands which is the binding of several subchannels, the number of IEs decreases as shown in Fig. 1. Under our assumption that all the subchannels are independent and identical, the probability for selecting user $k$ in one subband, $P r_{k}$, is all the same for all users, $1 / K$, where $K$ is the number of total users. Therefore, the number of IEs is derived as follows, where $N$ is the total number of subchannels and $p$ is the subband size.

$$
E[N o \text { of } I E]=\sum_{k=1}^{K} \frac{N}{p} \cdot P r_{k}=\frac{N}{p}
$$

Let $r$ be the constant value for transforming units from bit to sec, which influences on the modulation order and coding rate. If we ignore the fixed number of bits of DL-MAP, we can obtain $T_{M A P}$, the MAP transmission time by multiplying the number of IE by constant $r$. Since one frame length, $T_{\text {frame }}$, can be divided into overhead transmission time and data transmission time, the data transmission time, $T_{\text {data }}$, is calculated by subtracting $T_{M A P}$ from $T_{\text {frame }}$. Therefore, we can derive the total throughput as the product form of $E[M]$ and $T_{\text {data }}$. We can get the optimal subband size $p^{*}$ to maximize the system throughput.

$$
p^{*}=\underset{p}{\arg \max }\left(\mu_{x}+\frac{\sqrt{2 \sigma_{x}^{2} \log K}}{p}\right) \cdot\left(T_{\text {frmae }}-\frac{N}{p} \cdot r\right)
$$

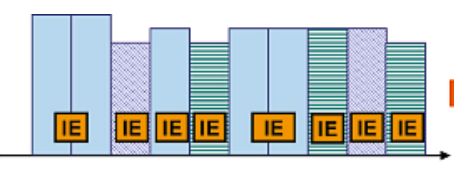

(a)

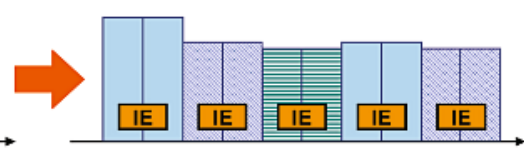

(b)

Fig. 1. Comparision of the number of IEs using (a) subband size=1 and (b) subband size $=2$ 


\subsection{Different Capacity of Each User Case}

In the previous subsection, we showed that optimum subband size in the case of i.i.d. subchannel. We now present numerical results in the more practical case where each user's subchannel has a different capacity distribution from other user's subchannel capacity. In this situation, it is advantageous to determine subband sizes that are different from each other. Let us assume $p_{k}$ is a subband size of user $k$, and $Y_{k}$ is the subband capacity of user $k, Y_{k}=$ $\operatorname{mean}\left\{X_{k, 1}, X_{k, 2}, \cdots, X_{k, p_{k}}\right\}$. Then, we can obtain the multiuser diversity gain as follows $[6]$.

$$
\begin{aligned}
& E[M]=\sum_{k=1}^{K} E\left[Y_{k} \mid Y_{k} \text { is maximal }\right] \\
& =\sum_{k=1}^{K}\left\{\int_{-\infty}^{\infty} y \frac{\operatorname{Pr}\left(Y_{k} \text { is maximal } \mid Y_{k}=y\right)}{\operatorname{Pr}\left(Y_{k} \text { is maximal }\right)} \frac{1}{\sqrt{2 \pi} \sigma_{k} / p_{k}} e^{-\frac{\left(y-\mu_{k}\right)^{2}}{2\left(\sigma_{k} / p_{k}\right)^{2}}} d y\right\} \\
& =\sum_{k=1}^{K}\left[\frac{1}{\operatorname{Pr}{ }^{\prime}} \int_{-\infty}^{\infty} \frac{y}{\sqrt{2 \pi} \sigma_{k} / p_{k}} e^{-\frac{\left(y-\mu_{k}\right)^{2}}{2\left(\sigma_{k} / p_{k}\right)^{2}}} \prod_{\substack{i=1 \\
i \neq k}}^{K}\left\{0.5+0.5 \operatorname{erf}\left(\frac{y-\mu_{i}}{\sqrt{2} \sigma_{i} / p_{i}}\right)\right\} d y\right]
\end{aligned}
$$

Alike notation of previous section, $M$ is the random variable of the selected user who has the largest subband capacity, and $\mu_{k}, \sigma_{k}^{2}$ represents the mean and variance value of user $k$ ' subchannel capacity respectively. Then, data transmission time is like below.

$$
T_{\text {data }}=T_{\text {frame }}-\left(\sum_{i=1}^{K} \frac{N}{p_{i}} \cdot \underset{i}{\operatorname{Pr}}\right) \cdot r
$$

Given (6) and (7), the total throughput of this system is given by product of $E[M]$ and $T_{d a t a}$, where $P r_{k}$ is defined as the probability which user $k$ is selected. $\mathrm{Pr}_{k}$ can be written as

$$
\begin{aligned}
& \operatorname{Pr}_{k}(\mu, \sigma)=\operatorname{Pr}\left(Y_{k} \text { is maximal }\right) \\
& =\int_{-\infty}^{\infty} \frac{1}{\sigma_{k} / p_{k} \sqrt{2 \pi}} e^{-\frac{\left(x-\mu_{k}\right)^{2}}{2\left(\sigma_{k} / p_{k}\right)^{2}}} \prod_{\substack{i=1 \\
i \neq k}}^{K}\left(0.5+0.5 \operatorname{erf}\left(\frac{x-\mu_{i}}{\sqrt{2} \sigma_{i} / p_{i}}\right)\right) d x
\end{aligned}
$$

To find the optimum solution which maximizes the total throughput, $\vec{p}^{*}=$ $\left[p_{1}^{*}, p_{2}^{*}, \ldots, p_{K}^{*}\right]$, is difficult because it is a non-linear optimization problem. Therefore, obtaining an optimal solution is very difficult. That is why we proposed heuristic algorithm MAP Reduced Resource Allocation (MRRA)

\subsection{MAP Reduced Resource Allocation}

We assume the Gaussian MIMO channel capacity with mean vector $\vec{\mu}=$ $\left[\mu_{1}, \mu_{2}, \ldots, \mu_{K}\right]$ and standard deviation vector $\vec{\sigma}=\left[\sigma_{1}, \sigma_{2}, \ldots, \sigma_{K}\right]$. In the MRRA algorithm, pre-calculated values of the optimum subband size according to the 
capacity distribution are used. We consider the i.i.d. channel assumption in order to simplify the problem. Namely, we assume that other users' channel capacity are the same as user $k$, and then calculated the optimum subband size of user $k, p_{k}$.

$$
p_{k}=\underset{p}{\arg \max }\left(\mu_{k}+\frac{\sqrt{2 \sigma_{k}^{2} \log K}}{p}\right) \cdot\left(T_{\text {frame }}-\frac{N}{p} \cdot r\right)
$$

We can determine the optimum subband size using (9). It is effective because the multi-dimensional search is not necessary. In addition, the adaptive scheme according to various capacity distributions can be exploited. However, if the subband size of each user is different from each other, a problem may occur in the resource allocation steps since the general resource allocation schemes are based on the same allocation unit size. Therefore, we consider integrating each user's subband size to one value by averaging. We have also proved the performance of this heuristic algorithm by simulation.

\section{Simulation Results}

We now describe some simulation experiments which were conducted to quantify the performance gains from proposed subband size determining scheme. Consider a 4x4 MIMO-OFDM system operating with $40 \mathrm{MHz}$ bandwidth and 256 subchannels under Rayleigh flat fading channel. Each time frame is $1 \mathrm{~ms}$ in length, and single-cell MIMO-OFDM system model is used. Let $\alpha$ be the initial MAP loading parameter that the proportional value of MAP message transmission time over a frame length. Accordingly, the initial MAP loading $\alpha$ is a real value between zero to one.

In order to evaluate the performance of the proposed MRRA scheme, it is compared with other resource allocation schemes. Here, we consider the comparison

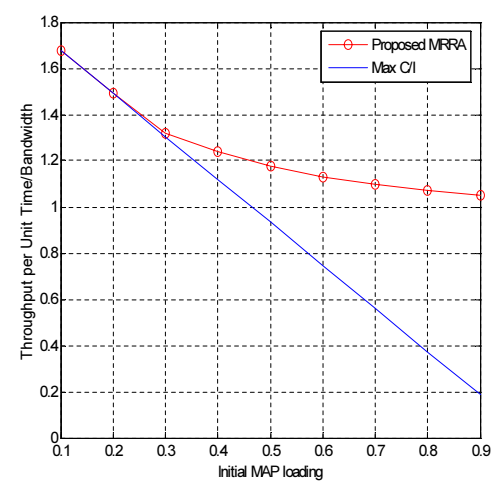

Fig. 2. Normalized throughput vs. subband size (i.i.d. case) 
with Max C/I resource allocation method. In the Max C/I resource allocation scheme, the system allocates a subband to the user who has the best channel capacity of that subband. The two schemes are compared via computer simulation using the same single-cell OFDM system model. Users' positions are all random, so that the capacity distribution of all users is different from each other.

Fig. 2 presents throughputs under the proposed MRRA scheme and max C/I scheme, respectively as the initial MAP loading increases. The proposed scheme achieves higher throughput when the MAP overhead get larger. Considering throughput, the MRRA scheme performs as high as $17 \%$ in the case of $\alpha=$ 0.5. In addition, when $\alpha$ is 0.8, the proposed MRRA scheme exhibits twice the throughput of Max C/I scheme. This throughput gain results from joint optimization of multiuser diversity and MAP transmission time.

In general, we observed that the performance gains from the MRRA scheme depend on the initial MAP loading. When there is heavy initial MAP loading, proposed scheme may help considerably improve performance.

\section{Conclusions}

In this paper, we have considered maximizing downlink throughput for MIMOOFDM systems by exploiting joint optimization between multiuser diversity and MAP overhead. In particular, we focused on determining the optimum subband size to maximize the system throughput. Simulation results show that the proposed MRRA scheme outperforms a Max C/I scheme in terms of throughput due to the optimality of our scheme. In the future MIMO-OFDM systems, the proposed MRRA algorithm should take into account specific resource allocation scheme in order to guarantee the QoS, which needs further investigation.

\section{References}

1. IEEE 802.16-2004, "IEEE Standard for Local and Metropolitan Area Networks Part 16: Air Interface for Fixed Broadband Wireless Access Systems," Oct.01, 2004

2. Peter J Smith and Mansoor Shafi, "On the Gaussian Approximation to the Capacity of Wireless MIMO System," Proceedings of IEEE ICC 2002, pp. 406-410, New York, May 2002

3. G.J. Focshini, "Layered Space-Time Architecture for Wireless Communication in a Fading Environment When Using Multi-Element Antennas," Bell Labs Technical Journal, pp.41-59, Oct.1996.

4. V.L. Girko, "A Refinement of the Central Limit Theorem for Random Determinants," The-ory of Probability and its Application, vol.42, no.1, pp.121-129, 1997.

5. B.W. Hochwald, T.L.Marzetta, and V. Tarokh, "Multi-Antenna Channel-Hardening and its Implications for Rate Feedback and Scheduling," IEEE transactions on Information Theory, vol. 50, no.9, pp.1893-1909, Sep. 2004

6. Athanasios Papoulis and S. Unnikrishna Pillai, "Probability, Random Variables and Stochastic Processes," 4th ed. Mc Graw Hill 